\title{
Using Chaos Synchronization to Estimate the Largest Lyapunov Exponent of Nonsmooth Systems
}

\author{
ANDRZEJ STEFANSKI and TOMASZ KAPITANIAK* \\ Division of Dynamics, Technical University of Lodz, Stefanowskiego 1/15, 90-924 Lodz, Poland
}

(Received 15 April 1999)

\begin{abstract}
We describe the method of estimation of the largest Lyapunov exponent of nonsmooth dynamical systems using the properties of chaos synchronization. The method is based on the coupling of two identical dynamical systems and is tested on two examples of Duffing oscillator: (i) with added dry friction, (ii) with impacts.
\end{abstract}

Keywords: Chaos synchronization, Lyapunov exponents, Nonsmooth systems

\section{INTRODUCTION}

The estimation of Lyapunov exponents is one of the fundamental tasks in the studies of dynamical systems. Lyapunov exponents measure the exponential rates of divergence or convergence of nearby trajectories in state space. Theoretical studies of Oseledec [1] and numerical algorithm of Benettin et al. [2] and Wolf et al. [3] allow an easy estimation of the spectrum of Lyapunov exponents for smooth systems described by the known motion equation. If such equations for the system are not known or it is nonsmooth, the estimation of Lyapunov exponents is not straightforward. In recent years methods of the calculation of Lyapunov exponents for dynamical systems with discontinuities have been proposed in some papers $[3,4]$.
Many real engineering systems can be considered as discontinuous, i.e. the mechanical systems with dry friction or with impacts. The dry friction in any engineering systems often leads to the appearance of the phenomenon of stick-slip oscillations which also have a discontinuous nature.

In this paper we propose a method of estimation of the largest Lyapunov exponent using chaos synchronization $[5,6]$. Our method is motivated by Fujisaka and Yamada's theoretical studies [5]. They have found the linear dependence between the synchronization value of the coupling coefficient of two identical continuous dynamical systems and the largest value of Lyapunov exponent of such coupled systems. As an example they have used the Lorenz system. We have shown that this approach can be applied for nonsmooth dynamical systems as well.

* Corresponding author. 


\section{THEORETICAL BACKGROUND FOR ESTIMATION PROCEDURE}

Consider a dynamical system which consists of two identical $n$-dimensional subsystems coupled by oneto-one negative feedback mechanism with a pair of coupling coefficients $k_{x}$ and $k_{y}$. The first order differential equations describing such a system can be written as

$$
\begin{aligned}
& \dot{x}=f(x)+k_{x}(y-x), \\
& \dot{y}=f(y)+k_{y}(x-y),
\end{aligned}
$$

where $x, y \in \mathbf{R}^{n}$ and $k_{x, y}=\left[k_{x, y} ; k_{x, y} ; \ldots ; k_{x, y}\right]^{\mathrm{T}} \in$ $\mathbf{R}^{n} \geq 0$ is a coupling vector. Let us assume that for $k_{x}=k_{y}=0$ each system $\mathrm{d} x / \mathrm{d} t=f(x)$ and $\mathrm{d} y /$ $\mathrm{d} t=f(y)$ evolves on an asymptotically stable chaotic attractor $\boldsymbol{A}$.

In the first part of our considerations we assume that the values of the coupling coefficients are equal to zero $\left(k_{x}=k_{y}=0\right)$. In this case we obtain two separate, identical dynamical systems given by equations

$$
\begin{aligned}
& \dot{x}=f(x), \\
& \dot{y}=f(y) .
\end{aligned}
$$

The solutions of Eq. (2) starting from different initial conditions represent two independent trajectories on the attractor $\boldsymbol{A}$. If initial conditions are the same the evolution of both subsystems is identical and we have ideal synchronization $(x=y)$.

Now we introduce a new variable $z$ representing the state difference between both subsystems during the time evolution. This variable is defined by the expression

$$
z=x-y,
$$

where $z \in \mathbf{R}^{n}$. In further analysis to simplify the notation and allow for the visualization we took $n=3$ and assumed that the evolution on the attractor $\boldsymbol{A}$ is characterized by one positive Lyapunov exponent. The absolute value of state difference

$$
q=\|\vec{z}\|=|x-y|
$$

describes the norm $\left(q \in \mathbf{R}^{1} \geq 0\right)$ of the state difference vector

$$
\vec{z}=\vec{x}-\vec{y} .
$$

The image of the vector equation (Eq. (5)) in phase space is shown in Fig. 1. The state difference vector is fixed to the end of vector $\vec{y}$ and its end is in touch with the end of vector $\vec{x}$ (see Fig. 1). The time evolution of the state difference vector $\vec{z}$ is defined in $z_{i}$ system of coordinates and can be determined as a sum of its $\vec{z}_{i}$ contributions in all directions of phase space (see Fig. 2):

$$
\vec{z}=\sum_{i=1}^{n} \vec{z}_{i}
$$

The origin of this system is fixed to the origin of vector $\vec{z}$. The axes of $z_{i}$-directions are parallel to the appropriate axes of phase space.

The time variations of the norm of state difference vector are determined by first time derivative of Eq. (4):

$$
\begin{array}{ll}
\dot{q}=\dot{x}-\dot{y} & \text { for } x \geq y, \\
\dot{q}=\dot{y}-\dot{x} & \text { for } x<y,
\end{array}
$$

and after substituting Eq. (2) in (7) we obtain

$$
\begin{aligned}
& \dot{q}=f(x)-f(y) \quad \text { for } x \geq y, \\
& \dot{q}=f(y)-f(x) \quad \text { for } x<y .
\end{aligned}
$$

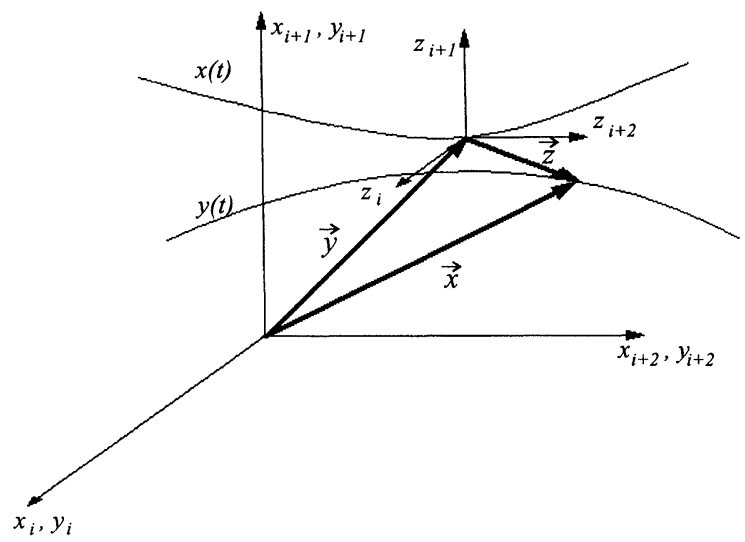

FIGURE 1 The image of the vector equation (Eq. (5)) in phase space. 


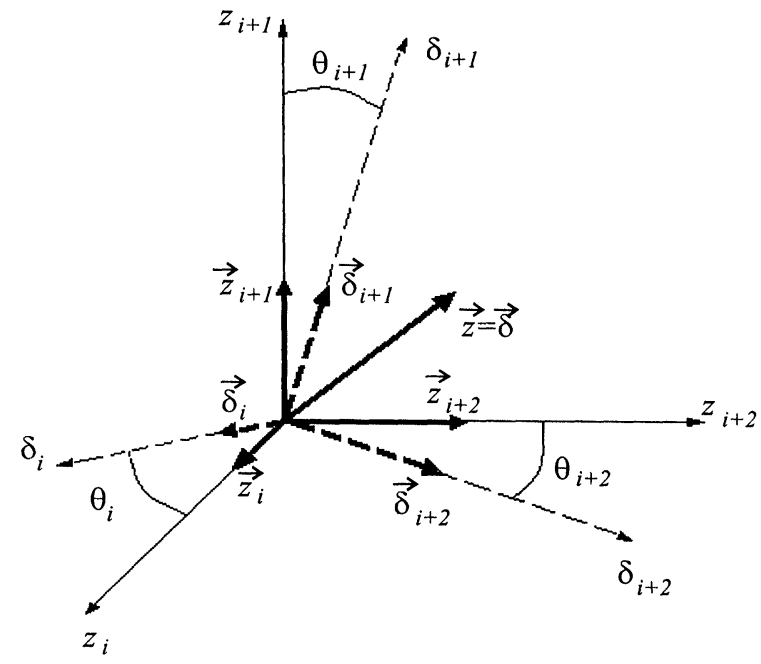

FIGURE 2 The spatial orientation of the system of coordinates associated with: (1) $\lambda$-distance vector (dotted line), (2) state difference vector (continuous line).

For small state difference vector, i.e. $q \ll|\boldsymbol{A}|$, where $|\boldsymbol{A}| \in \mathbf{R}^{1} \geq 0$ is the attractor size (maximum distance between two points on the attractor) in phase space, we can assume that the distance between trajectories of the subsystems under consideration is given by linearized equation resulting from definition of Lyapunov exponent

$$
\delta_{i}=\left|\delta_{i 0}\right| \mathrm{e}^{\lambda_{i} t},
$$

where $\lambda_{i}$ is a Lyapunov exponent, $\delta_{i}$ is the norm of the contribution $\vec{\delta}_{i}$ of $\lambda$-distance vector $\vec{\delta}$ in direction associated with $i$-number of Lyapunov exponent and $\delta_{i 0}$ is an initial distance in the same direction. The total $\lambda$-distance vector is a sum of contributions (see Fig. 2):

$$
\vec{\delta}=\sum_{i=1}^{n} \vec{\delta}_{i}
$$

The norm of this vector is given by the relation

$$
\delta=\|\vec{\delta}\|=\left[\sum_{i=1}^{n}\left(\delta_{i 0} \mathrm{e}^{\lambda_{i} t}\right)^{2}\right]^{1 / 2} .
$$

The Lyapunov exponents are related to the expanding or contracting nature of different directions in phase space. The spatial orientation of the coordinates system of directions associated with a given exponent varies in a complicated way during the evolution on the attractor. For that reason the axes of $z_{i}$-directions (state difference vector) are not covered with the axes associated with $\lambda$-directions ( $\lambda$-distance vector). Both systems of axes have the same origin and the angular difference between them is defined in each moment of time by angles $\theta_{i}(t)$ which depend on the position of trajectory on the attractor during the time evolution (see Fig. 2). The direction corresponding to exponent $\lambda_{i}=0$ is always tangential to phase trajectory.

From the above considerations the equality of state difference vector $\vec{z}$ and $\lambda$-distance vector $\vec{\delta}$ (for nearby orbits) arises:

$$
\vec{z}=\vec{\delta}
$$

To make further considerations easier we have introduced the following notations:

(1) $\lambda_{\max }$ - maximum Lyapunov exponent,

(2) $\delta_{0}$ - initial distance in $\lambda_{\max }$-direction,

(3) $\Delta \lambda_{j}$ - difference between $\lambda_{\max }$ and other Lyapunov exponent $\left(\Delta \lambda_{j}>0\right)$,

(4) $\lambda_{j}=\lambda_{\max }-\Delta \lambda_{j}-$ the rest of Lyapunov exponents,

(5) $\delta_{j 0}=m_{j} \delta_{0}$ - initial distance in $\lambda_{j}$-direction, $m_{j}$ is a constant value.

Putting the above substitutions in Eq. (11) we obtain

$$
\delta=\left[\delta_{0}^{2} \mathrm{e}^{2 \lambda_{\max } t}\left(1+\sum_{i=1}^{n-1} m_{j}^{2} \mathrm{e}^{-2 \Delta \lambda_{j} t}\right)\right]^{1 / 2} .
$$

During the time evolution the sum in Eq. (13) decreases to zero and the distance associated with $\lambda_{\max }$-direction becomes dominant. Hence, the norm of the $\lambda$-distance vector can be finally written in a reduced form:

$$
\delta=\left|\delta_{0}\right| \mathrm{e}^{\lambda_{\max } t}
$$

From the equality given by Eq. (12) the similar equality of the norms of both vectors results. 
It allows us to make a substitution:

$$
q=\left|\delta_{0}\right| \mathrm{e}^{\lambda_{\max } t} .
$$

The first time derivative of Eq. (15) is given by the relation

$$
\dot{q}=\lambda_{\max }\left|\delta_{0}\right| \mathrm{e}^{\lambda_{\max } t} .
$$

The comparison of Eq. (8) and (16) gives the equalities:

$$
\begin{array}{ll}
x \geq y: & f(x)-f(y)=\lambda_{\max }\left|\delta_{0}\right| \mathrm{e}^{\lambda_{\max } t} \\
x<y: & f(y)-f(x)=\lambda_{\max }\left|\delta_{0}\right| \mathrm{e}^{\lambda_{\max } t} .
\end{array}
$$

In the next step we introduce the nonzero coupling parameters $k_{x}$ and $k_{y}$ in Eq. (1). In this case the equation describing the first time derivative of the norm state difference vector assumes the following forms:

$$
\begin{array}{ll}
x \geq y: & \dot{q}=f(x)-f(y)-\left(k_{x}+k_{y}\right) q \\
x<y: & \dot{q}=f(y)-f(x)-\left(k_{x}+k_{y}\right) q .
\end{array}
$$

Using Eq. (17) in (18) we obtain:

$$
\frac{\mathrm{d} q}{\mathrm{~d} t}=\lambda_{\max }\left|\delta_{0}\right| \mathrm{e}^{\lambda_{\max } t}-\left(k_{x}+k_{y}\right) q .
$$

To separate variables we have to divide Eq. (19) by expression $q / \mathrm{d} t$ and next use the substitution $\left|\delta_{0}\right| \mid$ $q=\exp \left(-\lambda_{\max } t\right)$ arising from Eq. (15). Now Eq. (19) is given in the form:

$$
\frac{\mathrm{d} q}{q}=\left[\lambda_{\max }-\left(k_{x}+k_{y}\right)\right] \mathrm{d} t,
$$

and has the following solution:

$$
q=q_{0} \mathrm{e}^{\left[\lambda_{\max }-\left(k_{x}+k_{y}\right)\right]},
$$

where $q_{0}$ is a constant defined by the initial conditions. The synchronization between coupled subsystems of Eq. (1) takes place when the fixed point of state difference becomes a stable attractor, i.e. $z=Z_{\mathrm{s}}=0$. From Eq. (21) one finds that fulfilling the inequality

$$
\lambda_{\max }<k_{x}+k_{y},
$$

guarantees stability and then the norm of state difference vector decreases to zero.

According to Eq. (22) the synchronization of two identical systems is possible when the sum of coupling parameters is larger than the value of the largest Lyapunov exponent of these systems. If $\lambda_{\max }<0$ then both systems synchronize in a stable fixed point. For $\lambda_{\max }=0$ such systems have a periodic solution and the synchronization takes place just after a small coupling is introduced or even without coupling, when the excitations in both systems have the same phase. If $\lambda_{\max }>0$ we can observe a linear covering of two independent effects:

(1) exponential divergence of nearby trajectories associated with positive Lyapunov exponent,

(2) exponential convergence due to the introduced coupling.

The first of these phenomena occurs only in direct neighbourhood of the synchronized state where linear effects are dominant. The second one acts in the entire phase space. For that reason the described covering of both effects takes place only nearby the synchronized state.

The fulfilling of the inequality (Eq. (22)) causes that synchronization manifold $x=y$ becomes an attractor, i.e. the evolution of 6-dimensional dynamical system (Eq. (1)) is reduced to the attractor $\boldsymbol{A}$ located on $x=y$ manifold. In opposite case $\left(\lambda_{\max }>k_{x}+k_{y}\right)$ the manifold $x=y$ is a repeller and synchronization is impossible. In the remaining parts of this paper we give evidence that this property can be very useful in the estimation of Lyapunov exponents of the systems for which the straightforward approach $[2,3]$ is impossible.

\section{PRACTICAL ESTIMATION PROCEDURE}

Based on the above mentioned properties of chaos synchronization we have proposed a method of estimation of the largest Lyapunov exponent. This method is independent of the type of dynamical 
system because it is not necessary to assume continuous character of expressions $f(x)$ and $f(y)$ to obtain synchronization of $\mathrm{d} x / \mathrm{d} t=f(x)$ and $\mathrm{d} y /$ $\mathrm{d} t=f(y)$ subsystems. In this paper we have concentrated on the application of our method for finding the largest Lyapunov exponent of dynamical systems with discontinuities.

To simplify the procedure we have assumed unidirectional coupling in Eq. (1). In this case one of the coupling coefficients is equal to zero (say, $k_{x}=0, k_{y}=k$ ) and Eq. (1) is reduced to the following form:

$$
\begin{aligned}
& \dot{x}=f(x), \\
& \dot{y}=f(y)+k(x-y) .
\end{aligned}
$$

Now the condition of synchronization (Eq. (22)) is given by the relation

$$
\lambda_{\max }<k .
$$

The smallest value of the coupling coefficient $k$, for which the synchronization takes place $k_{\mathrm{s}}$ is equal to the maximum Lyapunov exponent $\lambda_{\max }$.

To apply our method for any dynamical system it is necessary to build a double system with coupling according to Eq. (23). The next step is a numerical research of the synchronization parameter $k_{\mathrm{s}}$ for such augmented system. If the tested coupling parameter $k$ reaches the boundary value $k_{\mathrm{s}}$ then the largest Lyapunov exponent of investigated system amounts to $k_{\mathrm{s}}$.

The simplest way to find the value of $k_{\mathrm{s}}$ is making a bifurcation diagram of the state difference $z$ between coupled subsystems of Eq. (23) in function of tested coupling parameter $k$. An example of such a diagram is presented in Fig. 3. We can obtain the searched value of $k_{\mathrm{s}}$ as a point on the horizontal axis (coefficient $k$ ) where state difference $z$ reaches zero value.

The main problem which has been observed during practical applications of our method is a long time of transient motion before the system under consideration achieves the synchronized state. This problem appears near the boundary value $k_{\mathrm{s}}$.

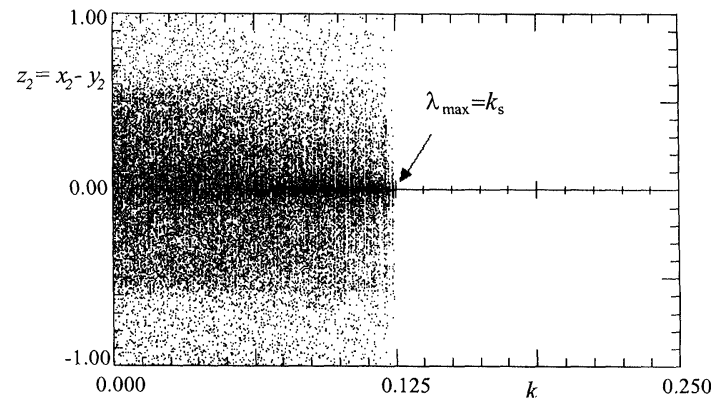

FIGURE 3 The bifurcation diagram showing state difference $z_{2}$ versus coupling coefficient $k$ for coupled Duffing oscillators (Eq. (27)) and estimated value of the largest Lyapunov exponent; $d=0.100$.

To make faster achievement of searched $k_{\mathrm{s}}$ value possible we have divided the phase space of the researched system into two regions. The first one is a direct neighbourhood of the synchronization subspace $x=y$. This region is bounded by a coefficient $\varepsilon$ which is a radius of $n$-dimensional sphere with a centre in the fixed point $Z_{\mathrm{s}}=0$ (Fig. 4(a)) or it can be considered as a radius of $n$-dimensional "corridor" surrounding the synchronization manifold (Fig. 4(b)). The second region is the remaining part of phase space. The way to achieve faster synchronization is to eliminate the long periods of time when phase trajectories evolute far away from synchronized state. For this purpose we have applied the method called elastic coupling which forces coupled systems to the evolution in the neighbourhood of $x=y$ manifold. The main idea of this method is the sudden jump of a coupling coefficient when state difference crosses the boundary $\varepsilon$ value. Inside the sphere $z \leq \varepsilon$ (Fig. 4(a)) the coupling parameter in Eq. (23) has tested $k$ value. Beyond the synchronization region $(z>\varepsilon)$ Eq. (23) assumes the following form:

$$
\begin{aligned}
& \dot{x}=f(x), \\
& \dot{y}=f(y)+K(x-y),
\end{aligned}
$$

where $K$ is the strong coupling parameter $(K \gg k)$ which does not allow the system evolving beyond $\varepsilon$-radius sphere. 


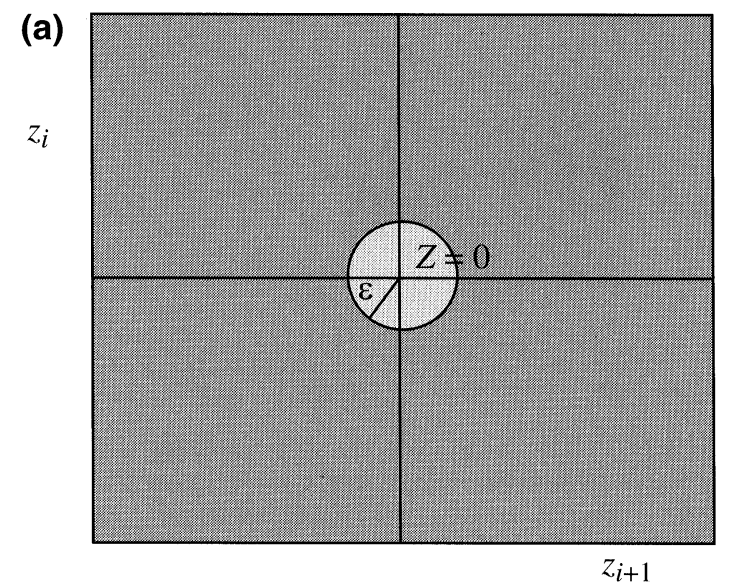

(b)


FIGURE 4 The idea of elastic coupling (see description in Section 3).

The proper values of $\varepsilon$ and $K$ can be elaborated in a way of individual studies of the given dynamical system.

\section{EXAMPLES}

In this section we present two types of dynamical systems with discontinuities. As an example of such system we have used the classical Duffing oscillator: (i) with added dry friction, (ii) with impacts.

\subsection{Duffing Oscillator with Dry Friction}

The equation describing the first example is as follows:

$$
\begin{aligned}
\dot{x}_{1}= & \dot{x}_{2}, \\
\dot{x}_{2}= & p \cos (\omega t)+c x_{1}\left(1-x_{1}^{2}\right)-h x_{2} \\
& -d\left(\frac{\mu_{0}-\mu_{1}}{1+\eta_{1}\left|x_{2}\right|}+\mu_{0}+\eta_{2} x_{2}^{2}\right) \operatorname{sign}\left(x_{2}\right) .
\end{aligned}
$$

The system (Eq. (26)) has been created by adding to the well known Duffing equation a term describing dry friction according to Popp-Stelter formula [7]. Its discontinuous nature makes any direct calculation of Lyapunov exponents difficult.

After putting the system under consideration (Eq. (26)) in (23) we obtain the augmented system in the following form:

$$
\begin{aligned}
\dot{x}_{1}= & x_{2}, \\
\dot{x}_{2}= & p \cos (\omega t)+c x_{1}\left(1-x_{1}^{2}\right)-h x_{2} \\
& -d\left(\frac{\mu_{0}-\mu_{1}}{1+\eta_{1}\left|x_{2}\right|}+\mu_{0}+\eta_{2} x_{2}^{2}\right) \operatorname{sign}\left(x_{2}\right), \\
\dot{y}_{1}= & y_{2}+k\left(x_{1}-y_{1}\right), \\
\dot{y}_{2}= & p \cos (\omega t)+c y_{1}\left(1-y_{1}^{2}\right)-h y_{2} \\
& -d\left(\frac{\mu_{0}-\mu_{1}}{1+\eta_{1}\left|y_{2}\right|}+\mu_{0}+\eta_{2} y_{2}^{2}\right) \operatorname{sign}\left(y_{2}\right) \\
& +k\left(x_{2}-y_{2}\right) .
\end{aligned}
$$

In our numerical simulations we have assumed the following values of parameters: $c=1.00, h=$ $0.25, \quad p=0.30, \quad \omega=1.00, \quad \eta_{1}=1.42, \quad \eta_{2}=0.005$, $\mu_{0}=0.25, \mu_{1}=0.05$.

Then the largest value of Lyapunov exponent of system (Eq. (26)) has been determined according to the way described in Section 3. The results of calculations are given by the graph shown in Fig. 5 which presents the changes of this exponent as a function of $d$ parameter representing amplifying dry friction force.

The bifurcation diagram of system (Eq. (26)), shown in Fig. 6 presents the dependence of velocity $x_{2}$ on $d$ parameter value. The $d$ parameter range is identical to that in Fig. 5. We can see bands when chaotic motion occurs (see Fig. 6) when Lyapunov 


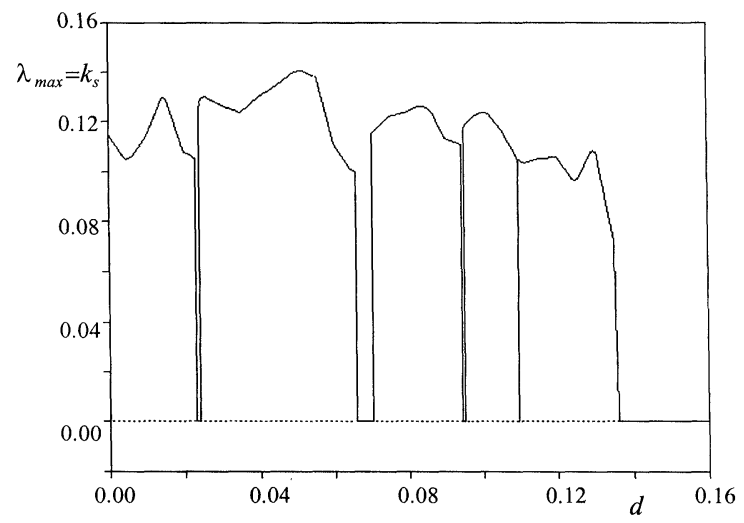

FIGURE 5 The largest Lyapunov exponent $\lambda_{\max }$ versus $d$ parameter value for the investigated Duffing oscillator with dry friction (Eq. (26)).

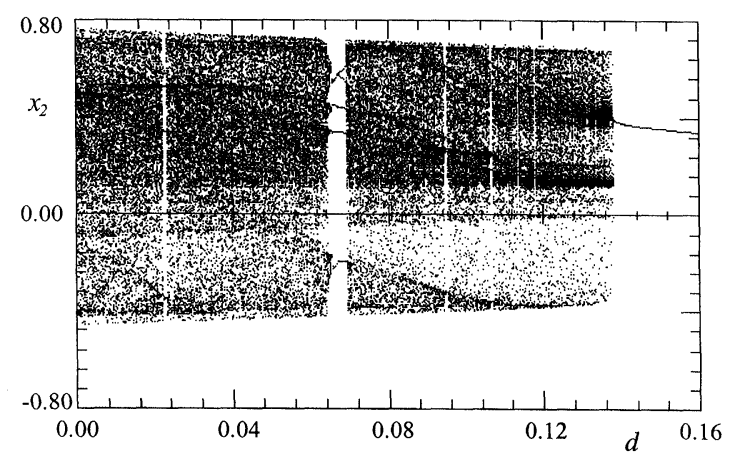

FIGURE 6 The bifurcation diagram of velocity $x_{2}$ versus $d$ parameter for Duffing oscillator with dry friction (Eq. (26)).

exponent takes positive value (Fig. 5). Also periodic motion occurs when zero value of Lyapunov exponent is found in Fig. 5. These results support our hypothesis that the values of the largest Lyapunov exponents obtained as above for systems with dry friction can agree with real ones (impossible to determine analytically) with high approximation.

\subsection{Duffing Oscillator with Impacts}

As the second example of discontinuous system we present again Duffing oscillator but now with impacts. The equations describing such a system can be written in forms:

$$
\begin{aligned}
\left|x_{1}\right|<X_{0}: & \dot{x}_{1}=x_{2} \\
\dot{x}_{2} & =p \cos (\omega t)+c x_{1}\left(1-x_{1}^{2}\right)-h x_{2}, \\
\left|x_{1}\right| \geq X_{0}: & x_{2 a}=-R x_{2 b},
\end{aligned}
$$

for two opposite buffers, and

$$
\begin{aligned}
x_{1}<X_{0}: \quad \dot{x}_{1} & =x_{2} \\
\dot{x}_{2} & =p \cos (\omega t)+c x_{1}\left(1-x_{1}^{2}\right)-h x_{2}, \\
x_{1} \geq X_{0}: & x_{2 a}=-R x_{2 b},
\end{aligned}
$$

for single buffer.

In the above equations $X_{0}$ is a position of the buffer, $R$ is the coefficient of restitution, $x_{2 b}$ is the velocity in a moment before impact and $x_{2 a}$ is the velocity just after impact.

Impact motion has a bit more complicated discontinuous nature than the motion of the system in the previous example. In phase space of the system with impacts the simultaneous existence of different types of attractors is possible. For that reason to determine the largest Lyapunov exponents of the systems (Eqs. (28) and (29)) we have applied the above mentioned method called elastic coupling.

We have estimated the largest Lyapunov exponents of systems under consideration for chosen values of the parameters. The results of numerical calculations are presented in Figs. 7 and 8 which show the phase portraits and associated with them the largest Lyapunov exponents (on the top of picture) which have been obtained using the described synchronization method.

We can see that chaotic motion occurs by positive Lyapunov exponents. It has also been confirmed (Figs. 7(a) and 8(a)) that synchronization of periodic systems can appear without coupling $\left(k_{\mathrm{s}}=\lambda_{\max }=0\right)$ between tested systems when excitation has the same phase. 
(a)

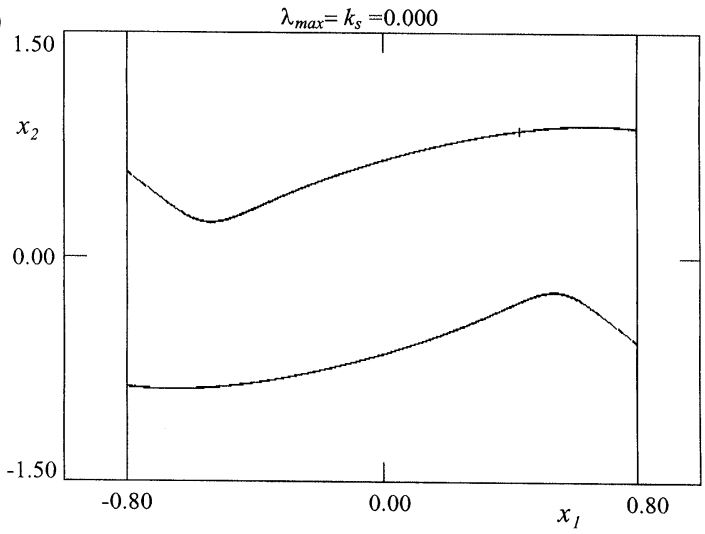

(b)

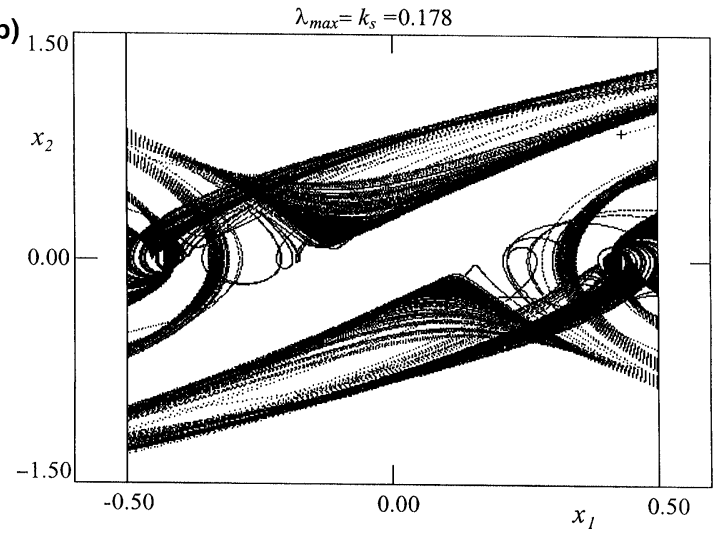

FIGURE 7 The phase portraits presenting the solutions of Duffing oscillator with impacts (two buffers - Eq. (28)) and the largest Lyapunov exponent $\lambda_{\max }$ associated with them: (a) periodic solution $-c=1.00, h=0.80, p=1.00, \omega=1.00$, $X_{0}=0.80, R=0.65$; (b) chaotic solution $-c=1.00, h=0.10$, $p=1.00, \omega=1.00, X_{0}=0.50, R=0.65$.

\section{CONCLUSIONS}

The present method allows us to estimate the value of the largest Lyapunov exponent of nonsmooth systems based on the properties of chaos synchronization. The developed method will be useful in quantifying, predicting and understanding chaos in nonsmooth discontinuous systems for which the straightforward calculation of the Lyapunov exponents is not possible. The approach presented in this paper can be generalized to the higher dimensional $(n>3)$ systems. This method can be applied for both numerical and experimental estimation of the largest Lyapunov exponent. In the first case one has (a)



(b)

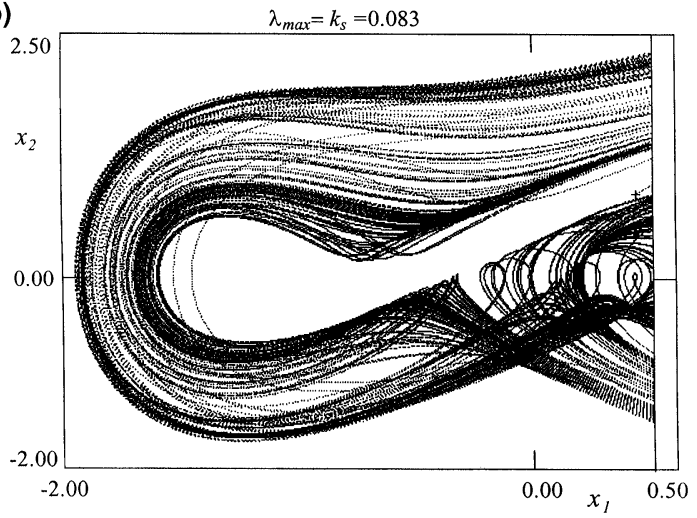

FIGURE 8 The phase portraits showing the solutions of Duffing oscillator with impacts (single buffer - Eq. (29)) and the largest Lyapunov exponent $\lambda_{\max }$ associated with them: (a) periodic solution $-c=1.00, h=0.80, p=1.00, \omega=1.00$, $X_{0}=0.50, R=0.65$; (b) chaotic solution $-c=1.00, h=0.10$, $p=1.00, \omega=1.00, X_{0}=0.50, R=0.65$.

to know the equation of motion. In the experimental case two examples of the system have to be created and coupled together.

\section{References}

[1] V.I. Oseledec: A multiplicative ergodic theorem: Lyapunov characteristic numbers for dynamical systems, Trans. Moscow Math. Soc. 19, 197-231 (1968).

[2] G. Benettin, L. Galgani, A. Giorgilli and J.-M. Strelcyn: Lyapunov exponents for smooth dynamical systems and Hamiltonian systems; a method for computing all of them, Part I: Theory; Part II: Numerical application, Meccanica 15, 9-20; 21-30 (1980).

[3] J.B. Wolf, H.L. Swift, Swinney and J.A. Vastano: Determining Lyapunov exponents from a time series, Physica D 16, 285-317 (1985). 
[4] P. Müller: Calculation of Lyapunov exponents for dynamical systems with discontinuities, Chaos, Solitons and Fractals 5(9), 1671-1681 (1995).

[5] H. Fujisaka and T. Yamada: Stability theory of synchronized motion in coupled-oscillator systems, Progress of Theoretical Physics 69(1), 32-47 (1983).
[6] L.M. Pecora and T.L. Carroll: Synchronization of chaos, Physical Review Letters 64, 221-224 (1990).

[7] K. Popp and P. Stelter: Nonlinear oscillations of structures induced by dry friction, in: Non-linear Dynamics in Engineering Systems, Ed. W. Schiehlen, Springer, New York (1990). 


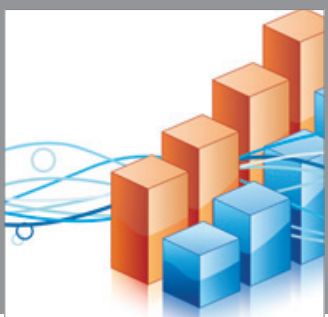

Advances in

Operations Research

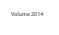

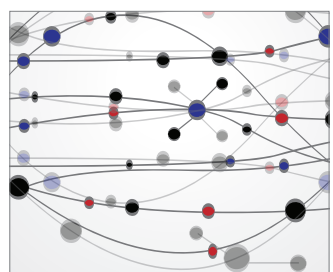

\section{The Scientific} World Journal
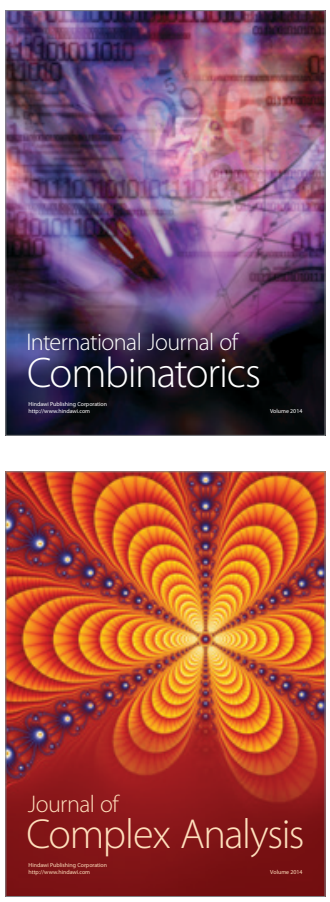

International Journal of

Mathematics and

Mathematical

Sciences
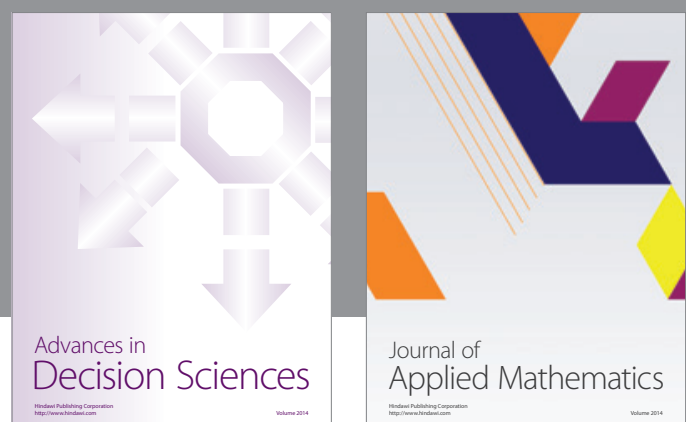

Journal of

Applied Mathematics


Submit your manuscripts at http://www.hindawi.com
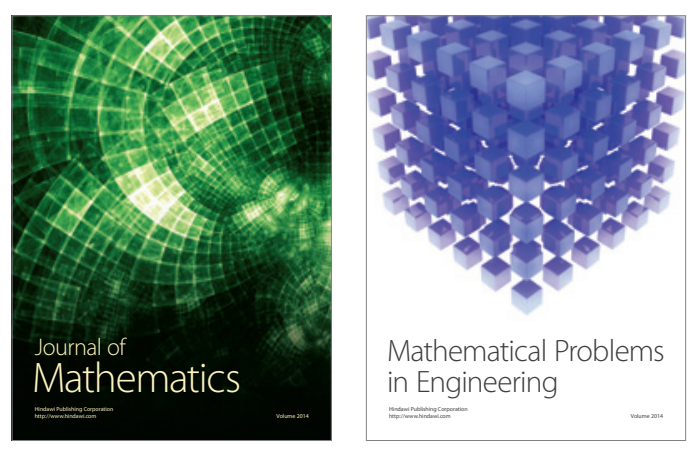

Mathematical Problems in Engineering
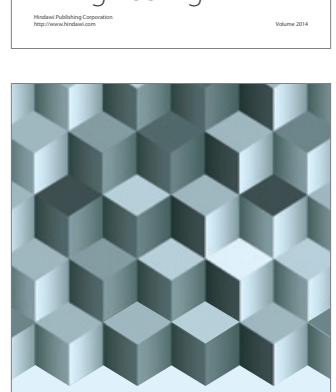

Journal of

Function Spaces
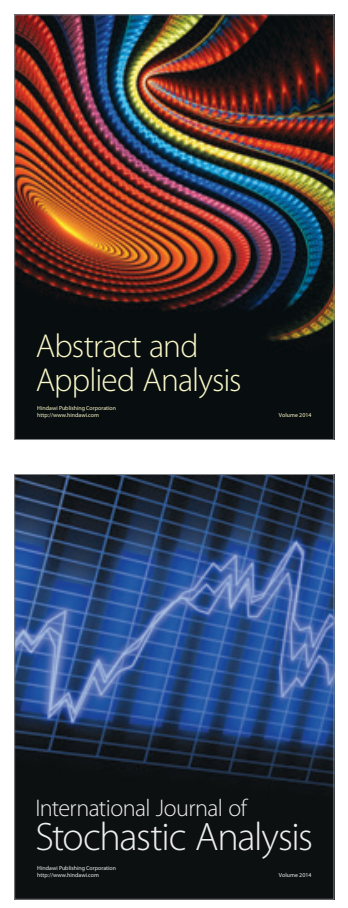

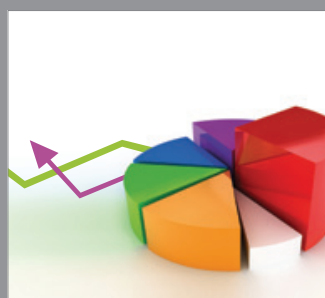

ournal of

Probability and Statistics

Promensencen
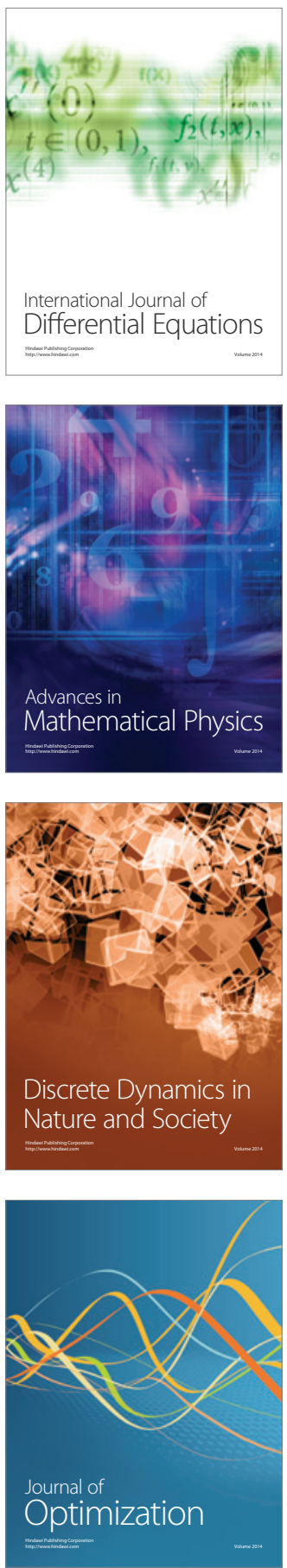